PROCEEDINGS OF THE

AMERICAN MATHEMATICAL SOCIETY

Volume 132, Number 8, Pages 2351-2356

S 0002-9939(04)07357-5

Article electronically published on January 20, 2004

\title{
CARLSON TYPE INEQUALITIES AND EMBEDDINGS OF INTERPOLATION SPACES
}

\author{
LEO LARSSON
}

(Communicated by N. Tomczak-Jaegermann)

AbStRaCt. We discuss the close relation between Carlson type inequalities

$$
\|a\|_{X} \leq K\|a\|_{A_{0}}^{1-\theta}\|a\|_{A_{1}}^{\theta}
$$

and interpolation, and prove embedding results for real interpolation spaces, in particular into weighted $L_{p}$-spaces.

\section{INTRODUCTION}

In 1934, F. Carlson [3] proved that the inequality

$$
\left(\sum_{n=1}^{\infty} a_{n}\right)^{4}<\pi^{2} \sum_{n=1}^{\infty} a_{n}^{2} \sum_{n=1}^{\infty} n^{2} a_{n}^{2}
$$

holds for all nonzero sequences $\left\{a_{n}\right\}_{n=1}^{\infty}$ of nonnegative numbers. He also noted that the corresponding integral inequality

$$
\left(\int_{0}^{\infty} f(x) d x\right)^{4} \leq \pi^{2} \int_{0}^{\infty} f^{2}(x) d x \int_{0}^{\infty} x^{2} f^{2}(x) d x
$$

holds. Carlson's inequalities (11) and (21) have been generalized in various directions and applied in several areas (see, e.g., [1, 6] and [7, and the references given there). In general, by a Carlson type inequality, we shall mean an inequality of the form

$$
\|a\|_{X} \leq K\|a\|_{A_{0}}^{1-\theta}\|a\|_{A_{1}}^{\theta}
$$

where $X, A_{0}$ and $A_{1}$ are normed spaces, $0<\theta<1$, and the constant $K$ is independent of $a$. We assume, here and hereafter, that $A_{0}$ and $A_{1}$ are embedded in a Hausdorff topological vector space.

The inequality (3) is equivalent to $X$ being of class $\mathcal{C}_{J}\left(\theta ; A_{0}, A_{1}\right)$; that is, there is a constant $K$ such that for all $t>0$

$$
\|a\|_{X} \leq K t^{-\theta} J\left(t, a ; A_{0}, A_{1}\right) .
$$

Here, $J$ is the Peetre $J$-functional, defined for all $a \in A_{0} \cap A_{1}$ by

$$
J\left(t, a ; A_{0}, A_{1}\right)=\max \left\{\|a\|_{A_{0}}, t\|a\|_{A_{1}}\right\}, \quad t>0 .
$$

Received by the editors December 11, 2002 and, in revised form, April 30, 2003.

2000 Mathematics Subject Classification. Primary 46B70; Secondary 46E30, 26 D15.

Key words and phrases. Inequalities, real interpolation spaces, Carlson's inequality, embeddings, weighted Lebesgue spaces. 
To see this equivalence, note that (4) can be written as

$$
\|a\|_{X} \leq K \max \left\{t^{-\theta}\|a\|_{A_{0}}, t^{1-\theta}\|a\|_{A_{1}}\right\} .
$$

The maximum of the two numbers above is smallest when

$$
t=\frac{\|a\|_{A_{0}}}{\|a\|_{A_{1}}}
$$

in which case it equals

$$
\|a\|_{A_{0}}^{1-\theta}\|a\|_{A_{1}}^{\theta} .
$$

Thus (5) holds precisely when (3) does.

If $X$ is complete, then $X$ is of class $\mathcal{C}_{J}\left(\theta ; A_{0}, A_{1}\right)$ if and only if we have the continuous embedding

$$
\left(A_{0}, A_{1}\right)_{\theta, 1} \subseteq X,
$$

where $\left(A_{0}, A_{1}\right)_{\theta, r}, 1 \leq r \leq \infty$, are the interpolation spaces arising from the real interpolation method (any of the equivalent $J$ - and $K$-methods). For a proof of this, see [2]. In the remainder of this paper, we discuss other embeddings of interpolation spaces; in particular, we obtain known and new embedding results for interpolation spaces into weighted $L_{p}$-spaces, but it is also discussed how even more general embeddings can be proved. More precisely, we investigate the embedding $\left(A_{0}, A_{1}\right)_{\theta, r} \subseteq X$ for $r>1$.

\section{The RESUlts}

We have the following embedding result.

Theorem 1. Let $(\Omega, \mu)$ be a measure space. Let the weights $\alpha, \alpha_{0}$ and $\alpha_{1}$ be defined on $\Omega$, and suppose that $\theta \in(0,1)$ and $p, p_{0}, p_{1} \in(0, \infty]$ are such that

$$
\frac{1}{q}:=\frac{1}{p}-\frac{1-\theta}{p_{0}}-\frac{\theta}{p_{1}} \geq 0 .
$$

Suppose, moreover, that there is a constant $C$ such that

$$
\mu\left(\left\{\omega \in \Omega ; 2^{m} \leq \frac{\alpha_{0}(\omega)}{\alpha_{1}(\omega)}<2^{m+1}\right\}\right) \leq C, m \in \mathbb{Z},
$$

and that

$$
\frac{\alpha}{\alpha_{0}^{1-\theta} \alpha_{1}^{\theta}} \in L_{s}(d \mu)
$$

for some $s \in[q, \infty]$. Let $X=L_{p}\left(\alpha^{p} d \mu\right)$ and $A_{i}=L_{p_{i}}\left(\alpha_{i}^{p_{i}} d \mu\right), i=0,1$. Then

$$
\left(A_{0}, A_{1}\right)_{\theta, p} \subseteq X .
$$

Remark 1. When $p=p_{\theta}$, defined as

$$
\frac{1}{p_{\theta}}=\frac{1-\theta}{p_{0}}+\frac{\theta}{p_{1}}
$$

the space $\left(A_{0}, A_{1}\right)_{\theta, p}$ is a weighted $L_{p}$-space. In general, however, this interpolation space can be complicated, and difficult to characterize (see, e.g., [8]). 
Proof of Theorem 1] Take $\theta_{i}, i=0,1$, such that $0<\theta_{0}<\theta<\theta_{1}<1$, and define

$$
\eta=\frac{\theta-\theta_{0}}{\theta_{1}-\theta_{0}}
$$

For $i=0,1$, let

$$
\beta_{i}=\alpha\left(\frac{\alpha_{0}}{\alpha_{1}}\right)^{\theta-\theta_{i}}
$$

and define $r_{i}$ by

$$
\frac{1}{r_{i}}=\frac{1}{q}+\frac{1-\theta_{i}}{p_{0}}+\frac{\theta_{i}}{p_{1}}
$$

Let $X_{i}=L_{r_{i}}\left(\beta_{i}^{r_{i}} d \mu\right), i=0,1$. By assumption,

$$
\frac{1}{r_{i}}-\frac{1-\theta_{i}}{p_{0}}-\frac{\theta_{i}}{p_{1}}=\frac{1}{q} \geq 0
$$

and

$$
\frac{\beta_{i}}{\alpha_{0}^{1-\theta_{i}} \alpha_{1}^{\theta_{i}}}=\frac{\alpha}{\alpha_{0}^{1-\theta} \alpha_{1}^{\theta}} \in L_{s}(d \mu)
$$

and so it follows by [6, Theorem 2] that there are constants $K_{i}$ such that

$$
\|f\|_{X_{i}} \leq K_{i}\|f\|_{A_{0}}^{1-\theta_{i}}\|f\|_{A_{1}}^{\theta_{i}} .
$$

In other words, $X_{i} \in \mathcal{C}_{J}\left(\theta_{i} ; A_{0}, A_{1}\right)$. By (the proof of) the Reiteration Theorem [2] Theorem 3.5.3], it follows that

$$
\left(A_{0}, A_{1}\right)_{\theta, p} \subseteq\left(X_{0}, X_{1}\right)_{\eta, p} .
$$

Now,

$$
\beta_{0}^{1-\eta} \beta_{1}^{\eta}=\alpha
$$

and

$$
\frac{1-\eta}{r_{0}}+\frac{\eta}{r_{1}}=\frac{1}{p}
$$

So [2, Theorem 5.5.1] implies that $\left(X_{0}, X_{1}\right)_{\eta, p}=X$. This completes the proof.

The scale of spaces $\left(A_{0}, A_{1}\right)_{\theta, r}$ is increasing with $r$. Thus (9) holds true if $p$ is replaced by any $r \in[1, p)$. However, the following partial converse to Theorem [ shows that we cannot, in general, go beyond $p$.

For each $t>0$, we define the Peetre $K$-functional on $A_{0}+A_{1}$ by

$$
K\left(t, f ; A_{0}, A_{1}\right)=\inf \left\{\left\|f_{0}\right\|_{A_{0}}+t\left\|f_{1}\right\|_{A_{1}} ; f=f_{0}+f_{1}, f_{i} \in A_{i}, i=0,1\right\} .
$$

Proposition 2. Given $p, p_{0}, p_{1} \in(0, \infty]$ and $\theta \in(0,1)$ satisfying (6), for any $r \in(p, \infty]$, there are a measure space $(\Omega, d \mu)$ and weights $\alpha, \alpha_{0}, \alpha_{1}$ satisfying (7) and (8) such that

$$
\left(A_{0}, A_{1}\right)_{\theta, r} \nsubseteq X \text {. }
$$

Proof. We consider $\Omega=(e, \infty)$ with the measure

$$
d \mu(x)=\frac{d x}{x}
$$

and define $\alpha(x)=1, \alpha_{0}(x)=x^{\theta}, \alpha_{1}(x)=x^{-(1-\theta)}$. Then the weights satisfy the hypotheses of Theorem 1 with $C=\log 2$ and $s=\infty$.

Let

$$
f(x)=\frac{1}{(\log x)^{1 / p}} .
$$


Then $f \notin X$. We want to show that $f \in\left(A_{0}, A_{1}\right)_{\theta, r}$. The norm on $\left(A_{0}, A_{1}\right)_{\theta, r}$ is given by

$$
\|f\|_{\theta, r}^{r}=\int_{0}^{\infty}\left(t^{-\theta} K\left(t, f ; A_{0}, A_{1}\right)\right)^{r} \frac{d t}{t},
$$

where $K$ is the Peetre $K$-functional, with the usual convention when $r=\infty$. If $0<t \leq e$, define $f_{0}^{(t)}=0$ and $f_{1}^{(t)}=f$. Then $\left\|f_{0}^{(t)}\right\|_{A_{0}}=0$, while

$$
\left\|f_{1}^{(t)}\right\|_{A_{1}}=\left(\int_{e}^{\infty} \frac{x^{-p_{1}(1-\theta)}}{(\log x)^{p_{1} / p}} \frac{d x}{x}\right)^{1 / p_{1}}=B .
$$

Thus

$$
\begin{aligned}
t^{-\theta} K\left(t, f ; A_{0}, A_{1}\right) & \leq t^{-\theta}\left\|f_{0}^{(t)}\right\|_{A_{0}}+t^{1-\theta}\left\|f_{1}^{(t)}\right\|_{A_{1}} \\
& =B t^{1-\theta} .
\end{aligned}
$$

If $t>e$, let $f_{0}^{(t)}=f \chi_{(e, t)}$ and $f_{1}^{(t)}=f \chi_{[t, \infty)}$. Then $f_{0}^{(t)}+f_{1}^{(t)}=f$ for all $t$. We have

$$
\begin{aligned}
\int_{\Omega}\left(f_{0}^{(t)} \alpha_{0}\right)^{p_{0}} d \mu & =\int_{e}^{t} \frac{x^{p_{0} \theta}}{(\log x)^{p_{0} / p}} \frac{d x}{x} \\
& \leq C_{0}^{p_{0}} \frac{t^{p_{0} \theta}}{(\log t)^{p_{0} / p}}
\end{aligned}
$$

and

so that

$$
\int_{\Omega}\left(f_{1}^{(t)} \alpha_{1}\right)^{p_{1}} d \mu \leq C_{1}^{p_{1}} \frac{t^{-p_{1}(1-\theta)}}{(\log t)^{p_{1} / p}}
$$

It follows that

$$
t^{-\theta}\left\|f_{0}^{(t)}\right\|_{A_{0}}+t^{1-\theta}\left\|f_{1}^{(t)}\right\|_{A_{1}} \leq \frac{C_{0}+C_{1}}{(\log t)^{1 / p}} .
$$

$$
\|f\|_{\theta, r}^{r} \leq B^{r} \int_{0}^{e} t^{(1-\theta) r} \frac{d t}{t}+\left(C_{0}+C_{1}\right)^{r} \int_{e}^{\infty} \frac{d t}{t(\log t)^{r / p}} .
$$

Since $r>p$, the last integral converges (and so does the first). It follows that $f \in\left(A_{0}, A_{1}\right)_{\theta, r}$.

For completeness, we give the following multi-dimensional version of Theorem 1 Theorem 3. Let $n$ be a positive integer, and let measure spaces $\left(\Omega^{(j)}, \mu^{(j)}\right), j=$ $1, \ldots, n$, be given. Suppose that weights $\alpha^{(j)}, \alpha_{0}^{(j)}$ and $\alpha_{1}^{(j)}$ are defined on $\Omega^{(j)}$, and that the parameters $\theta \in(0,1)$ and $p, p_{0}, p_{1} \in(0, \infty]$ satisfy

$$
\frac{1}{q}=\frac{1}{p}-\frac{1-\theta}{p_{0}}-\frac{\theta}{p_{1}} \geq 0 \text {. }
$$

Let $1 \leq k \leq n$, and suppose that there are constants $C_{j}, j=1, \ldots, k$, such that

$$
\mu^{(j)}\left(\left\{\omega_{j} \in \Omega^{(j)} ; 2^{m} \leq \frac{\alpha_{0}^{(j)}\left(\omega_{j}\right)}{\alpha_{1}^{(j)}\left(\omega_{j}\right)}<2^{m+1}\right\}\right) \leq C_{j}, m \in \mathbb{Z}
$$

and that

$$
\frac{\alpha^{(j)}}{\left(\alpha_{0}^{(j)}\right)^{1-\theta}\left(\alpha_{1}^{(j)}\right)^{\theta}} \in L_{s_{j}}\left(d \mu^{(j)}\right)
$$


where

$$
s_{j} \in[q, \infty] \text { and } \quad \frac{1}{s_{1}}+\cdots+\frac{1}{s_{k}} \geq \frac{k-1}{q} .
$$

Suppose also that

$$
\frac{\alpha^{(j)}}{\left(\alpha_{0}^{(j)}\right)^{1-\theta}\left(\alpha_{1}^{(j)}\right)^{\theta}} \in L_{q}\left(d \mu^{(j)}\right), j=k+1, \ldots, n .
$$

If

$$
A_{i}=L_{p_{i}}\left(\prod_{j=1}^{n} \alpha_{i}^{(j)} d \mu^{(j)}\right), i=0,1
$$

and

$$
X=L_{p}\left(\prod_{j=1}^{n} \alpha^{(j)} d \mu^{(j)}\right)
$$

then (9) holds.

Sketch of Proof. As in the proof of Theorem 1 we define the spaces $X_{i}, i=0,1$, and use [6, Theorem 5] to show that $X_{i} \in \mathcal{C}_{J}\left(\theta_{i} ; A_{0}, A_{1}\right)$. The desired embedding follows from the Reiteration Theorem and the fact that $X=\left(X_{0}, X_{1}\right)_{\eta, p}$.

\section{Concluding Remarks}

Remark 2. The proofs of Theorems 1 and 3 rely on the fact that we have a Carlson type inequality for the spaces involved, and that we can rewrite our space $X$ as an interpolation space and use the Reiteration Theorem. We can state the conclusion in more general terms; namely, as soon as we can prove a Carlson type inequality for the auxiliary spaces $X_{0}$ and $X_{1}$, or equivalently, can show that $X_{i}$ is of class $\mathcal{C}_{J}\left(\theta_{i} ; A_{0}, A_{1}\right), i=0,1$, we have the embedding

$$
\left(A_{0}, A_{1}\right)_{\theta, p} \subseteq\left(X_{0}, X_{1}\right)_{\eta, p} .
$$

The spaces $X$ and $A_{i}, i=0,1$, need not necessarily be weighted $L_{p}$-spaces, as long as we have a Carlson type inequality and a corresponding reiteration theorem. Much more general embeddings could be achieved using the methods of this paper.

Remark 3. Carlson type inequalities have previously been used in the interpolation context, for instance by N. Ya. Kruglyak, L. Maligranda and L.-E. Persson [5], who applied the Brundyı-Kruglyak construction, which was also used in the solution to the classical $K$-divisibility problem. Their version of the inequality involved "optimal blocks", and was used to characterize the Peetre interpolation functor \langle\rangle$_{\varphi}$. See also [4], where the \pm method was introduced.

\section{ACKNOWLEDGMENT}

The author would like to thank Professors Svante Janson and Lars-Erik Persson for invaluable comments and suggestions on pre-final versions of this work. 


\section{REFERENCES}

[1] Barza, S., Burenkov, V., Pečarić, J., and Persson, L.-E.: Sharp Multidimensional Multiplicative Inequalities for Weighted $L_{p}$ Spaces with Homogeneous Weights, Math. Inequal. Appl. 1 (1998), 53-67. MR 98k:26031

[2] Bergh, J. and Löfström, J.: Interpolation Spaces: An Introduction, Springer-Verlag, BerlinHeidelberg-New York, 1976. MR 58:2349

[3] Carlson, F.: Une inégalité, Ark. Mat. Astr. Fysik 25B (1934), No. 1.

[4] Gustavsson, J. and Peetre, J.: Interpolation of Orlicz Spaces, Studia Math. 60 (1977), 33-59. MR 55:11021

[5] Kruglyak, N. Ya., Maligranda, L., and Persson, L.-E.: A Carlson Type Inequality with Blocks and Interpolation, Studia Math. 104 (1993), 161-180. MR 94k:46153

[6] Larsson, L.: A New Carlson Type Inequality, Math. Inequal. Appl. 6 (2003), 55-79. MR 2003k:26026

[7] Levin, V. I.: Sharp Constants in Carlson Type Inequalities, Doklady Akad. Nauk, SSSR 59 (1948), 635-638 (Russian). MR 9:415b

[8] Maligranda, L. and Persson, L.-E.: Real Interpolation between Weighted $L^{p}$ and Lorentz Spaces, Bull. Polish Acad. Sci. Math. 35 (1987), No. 11-12, 765-778. MR 89m:46055

Department of Mathematics, Uppsala University, Box 480, SE-751, 06 Uppsala, SweDEN

E-mail address: leo@math.uu.se 